\title{
Debulking From Within: A Robotic Steerable Cannula for Intracerebral Hemorrhage Evacuation
}

\author{
Jessica Burgner, Member, IEEE, Philip J. Swaney, Student Member, IEEE, Ray A. Lathrop, Student Member, IEEE, \\ Kyle D. Weaver, and Robert J. Webster III*, Member, IEEE
}

\begin{abstract}
New approaches to intracerebral hemorrhage management are motivated by its high incidence and $40 \%$ mortality rate. Surgery is sometimes attempted to decompress the brain, although patient outcomes are similar regardless of whether surgery occurs. We hypothesize that surgical decompression is not more effective because current open surgical techniques disrupt healthy brain tissue to access the clot formed by the hemorrhage, offsetting the benefits of surgery. To address this, we propose a less invasive needle-based approach in which the clot is debulked from within using a superelastic, precurved aspiration cannula that is deployed from a needle. The tip of this aspiration cannula is controlled by coordinated insertion and retraction of the cannula and needle, as well as axial rotation of the cannula. We describe the design of a sterilizable and biocompatible robot that can control the three degrees of freedom of the needle and cannula. Image guidance is achieved by adapting an approach originally developed for brain biopsy. We provide an optimization method for the selection of the precurvatures of one or more sequentially used aspiration cannulas to maximize hemorrhage evacuation, based on preoperative medical image data. In vitro experiments demonstrate the feasibility of evacuating 83-92\% of hemorrhage volume, depending on the number of tubes and deployment method used.
\end{abstract}

Index Terms-Active cannula, concentric tube robot, continuum robot, minimally invasive neurosurgery, robot-assisted surgery.

\section{INTRODUCTION}

A PPROXIMATELY 1 in 50 people will have an intracerebral hemorrhage (ICH) at some point in their lives, and the one-month mortality rate is approximately $40 \%$ [1]. ICH occurs when a blood vessel in the brain ruptures and blood accumulates within the cranial cavity, compressing the brain. It may be

Manuscript received January 7, 2013; accepted April 25, 2013. Date of publication April 30, 2013; date of current version August 16, 2013. This work was funded by the National Science Foundation under Grant IIS-1054331 and a Graduate Research Fellowship. The work of J. Burgner was supported in part by a fellowship from the German Academic Exchange Service. Asterisk indicates corresponding author.

J. Burgner is with the Hannover Centre for Mechatronics, Leibniz Universität Hannover, D-30060 Hannover, Germany (e-mail: burgner@mzh. uni-hannover.de).

P. J. Swaney and R. A. Lathrop are with the Department of Mechanical Engineering, Vanderbilt University, Nashville, TN 37235 USA (e-mail: philip.j. swaney@vanderbilt.edu; Ray.A.Lathrop@vanderbilt.edu).

K. D. Weaver is with the Department of Neurological Surgery, Vanderbilt University Medical Center, Nashville, TN 37235 USA (e-mail: kyle.weaver@ vanderbilt.edu).

${ }^{*}$ R. J. Webster III is with the Department of Mechanical Engineering, Vanderbilt University, Nashville, TN 37235 USA and also with the Department of Neurological Surgery, Vanderbilt University Medical Center, Nashville, TN 37232 USA (e-mail: robert.webster@vanderbilt.edu).

Color versions of one or more of the figures in this paper are available online at http://ieeexplore.ieee.org.

Digital Object Identifier 10.1109/TBME.2013.2260860 treated with drugs or surgical evacuation via open craniotomy. While one would expect decompression via hemorrhage evacuation to result in improved patient outcomes, there is no clinical data supporting this for the majority of ICH patients. Benefits of various treatments have only been shown to date in patients selected for factors like small, superficial lesions and a good preoperative performance status [2]-[6], and there remains no treatment of proven clinical benefit for typical ICH patients [7]. In standard open surgical procedures, where the brain substance is cut with electrocautery, tubular retraction systems with or without endoscopic assistance and Archimedes screw-type devices have been applied, but with only minimal improvement in outcomes [3], [8], [9].

A proportion of this failure may be due to permanent injury by the hemorrhage, which is irreversible even with evacuation. However, it is generally believed by neurosurgeons that there is a volume of brain tissue that is a risk but which may be salvaged and returned to preinjury function if its conditions are optimized, and it is known that decompression can help to optimize brain conditions [10]. However, this is currently challenging to accomplish for all hemorrhage locations and particularly for deep hemorrhages. For many hematomas, an operative trajectory of any significant dimension would result in more tissue volume disruption than would be saved by the evacuation of the hematoma. This results in only superficial hematomas currently being candidates for evacuation and may be partially responsible for the lack of encouraging clinical data on the benefit of surgical decompression.

Although there have been several minimally-invasive surgical approaches proposed, such as locally delivered ultrasound with thrombolysis and endoscopic aspiration of the clot [11], [12], these have only been applied in small case studies and have not been adopted as the standard treatment for ICH. While it is possible that one of these new minimally-invasive approaches may ultimately succeed in solving the problem of ICH decompression, until one achieves widespread clinical use and is successful in reducing the high mortality and complication rates of $\mathrm{ICH}$, there is ample motivation to pursue new approaches such as the one we propose in this paper. We hypothesize that a robot that can access the site of the hemorrhage through a needle-sized entry path and then debulk the clot from within will reduce the trauma to healthy brain tissue currently associated with surgical decompression.

The problem of needle-based neurosurgery is not new in the field of surgical robotics. Indeed, the first surgical robot was used for this purpose [13], and much subsequent progress has been made on stereotactic robotic systems, as reviewed in [14]-[16]. 
These prior systems use straight needle trajectories with imageguidance to hit specific targets within the brain. The system described in this paper is similar to prior systems in its use of image-guidance to hit specific intracerebral targets, but different in its need for dexterous motion of the needle tip within the lesion. This desire for motion within the lesion was an early motivation for bevel-steered needles [17], [18], but maximum curvature and interventional tool integration challenges have thus far prevented ICH experiments from being conducted with bevel steering. Interventional tool manipulation within a hemorrhage was first addressed robotically through an entry path of endoscopic surgery dimensions when a straight morcellator was used to debulk a hematoma in the brain [19]. This study showed that while manual hematoma removal was faster, robotic hematoma removal resulted in less damage to the surrounding brain tissue in in vitro studies [19]. However, despite these promising results, robotic assistance has yet to become routinely used in treating ICH patients.

In this paper, we present a needle-based robotic system for image-guided evacuation of ICHs that is no more invasive than a standard brain biopsy, yet enables ICH clots to be debulked from within via articulation of an aspiration cannula that extends from the needle tip. We note that such a robot system does not need to debulk the entire clot [20]. Based on the judgment of experienced neurosurgeons, we anticipate clinically meaningful decompression to begin at approximately $25-50 \%$ removal of the clot volume, though future clinical studies will be needed to verify this estimate.

Our system uses a simple two-tube, 3 degree-of-freedom (DOF) concentric tube robot, also known as an active cannula [21], [22], which consists of a straight, stiff outer needle and an inner curved superelastic cannula. In contrast to bevel steered needles, the active cannula does not depend on tissue interaction to achieve steering. In addition, the combination of tubes enables straight entry pathways toward the hemorrhage and high curvatures within the clot for dexterous aspiration. In prior tube property design research for these robots, it has been assumed that the robot will consist of multiple tubes (usually at least 3) whose properties (precurvatures, stiffnesses, etc.) must be selected a priori based on task requirements and then remain fixed throughout use of the robot [23]-[26].

Here, we consider a somewhat different paradigm for design and use of these robots, namely that tubes can be added and removed from the robot during surgery. Thus, in ICH evacuation, the clot is debulked not by one complex concentric tube robot, but by what can be thought of as multiple simple concentric tube robots, used sequentially. A preliminary version of some results in this manuscript can be found in [27]. This archival version combines the results in [27] with additional material that comprises the entirety of Section III, as well as a new patient case study (Section IV) and new experimental results (Section V-B). In the sections that follow, we describe the design of a biocompatible actuation unit to control the needle and cannula, an image-guidance protocol, tube design algorithms, and in vitro experiments illustrating the feasibility of evacuating hematoma phantoms matching the geometry of $\mathrm{ICH}$ patient cases.

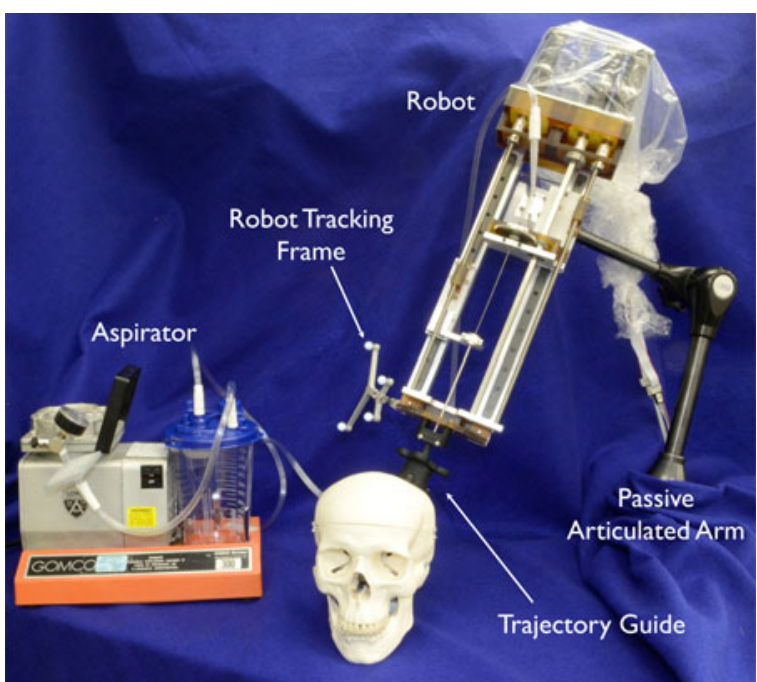

Fig. 1. Robot-assisted ICH evacuation prototype setup attached to a phantom skull. The robot is positioned and held in place with a passive articulated arm. The aspirator is attached to the aspiration cannula that enters the brain through a trajectory guide attached to the skull. A reference frame is rigidly attached to the robot for optical tracking.

\section{ROBOT SYSTEM}

The ICH robot system is shown in Fig. 1. It includes a sterilizable robotic actuation unit capable of controlling a two-tube active cannula, a head-mounted trajectory guide to enable imageguided targeting of the hemorrhage, a passive articulated arm (MA60003, NOGA, USA), and an aspirator to evacuate the hemorrhage. The robot controls 3 DOF of two tubes. The outer tube (referred to as "the needle" in the introduction) is a straight, stiff tube made of stainless steel. The inner aspiration cannula is a tube that is precurved and made of superelastic nitinol. As it passes through the outer tube, it is straightened, but it returns to its precurved shape when it exits the tip of the outer tube. The actuation unit controls insertion and retraction of both tubes, and axial rotation of the inner tube.

\section{A. Sterilizable, Biocompatible, Robotic Actuation Unit}

The robotic actuation mechanism was designed to be both sterilizable and biocompatible. Safety, ease of use, and streamlined integration into the surgical workflow were also primary considerations. Most prior actuation units for concentric tube robots have been research lab prototypes, and sterilization and workflow considerations have been left to future work. The exception is the work of Graves et al., who suggested a fully disposable approach in which the entire robot is thrown away after each surgery [28]. A sterilizable manual version without motors was also described in [29]. It remains to be seen whether surgeons can learn to use a system like ours sufficiently well for a manual approach to be viable (we suspect not), and economic considerations will likely dictate the choice between the disposable or reusable paradigm in an eventual commercial system, though both approaches have merit. Our design contribution here is the first reusable, sterilizable, and operating room ready actuation unit for concentric tube robots. It is constructed 


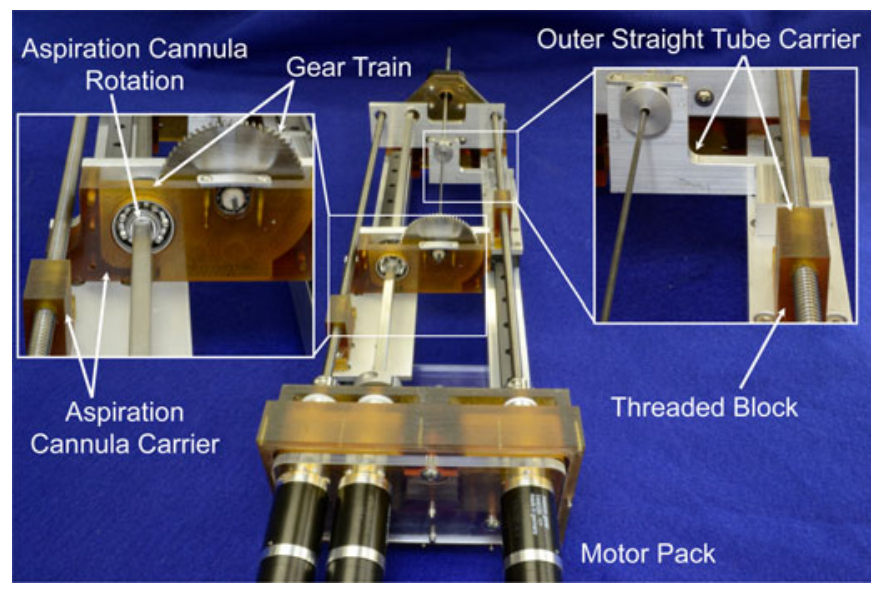

Fig. 2. Two carriers are translated via threaded blocks using lead screws and slide along a linear track. One carrier translates the straight outer tube, and the second carrier translates the precurved aspiration tube, which is rotated with a square shaft attached to a small gear that meshes with the large gear attached to the tube hub.
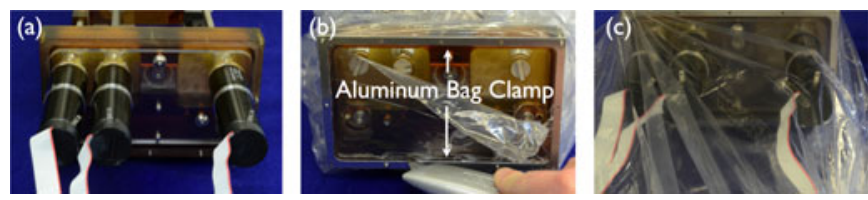

Fig. 3. Sterile barrier concept. (a) Motor plate is removed from the robot before sterilization. (b) Sterile bag is attached to the sterile robot using an aluminum clamp, enabling the motor plate to be subsequently attached and the motors to engage with the Oldham couplings (see Fig. 4). (c) Motor plate is reattached and the bag pulled over it. The robot is now sterile.

entirely from autoclavable and biocompatible components, with a modular motor pack that can be bagged.

The actuation unit mechanism is depicted in Fig. 2. The straight outer tube is inserted and retracted using a lead screw that acts on the tube's carrier, which rides on a linear slide. The same concept applies to the second carrier, which actuates the inner aspiration tube. Rotary motion is transmitted to the tube from the motor pack using a square shaft that interfaces with a gear train (see Fig. 2). Both lead screws and the square shaft are actuated by motors housed in the motor pack at the end of the robot furthest from the patient. Three motors are mounted on a motor plate that can easily be attached and detached from the robot.

The motors are isolated from the sterile field by a tortuous path sterility barrier with Oldham couplings that transmit rotational motion through a sterile bag (see Figs. 3 and 4). To set up the robot in the operating room, all components except the motor pack are first autoclave sterilized with the motor pack detached. A sterile bag is attached to the robot using an aluminum bag clamp, followed by attachment of the motor plate. The sterile bag is then pulled over the motor pack.

The tubes of the active cannula are bonded to their respective hubs using biocompatible and autoclavable glue (Loctite, M-21 HP). Example tubes are shown in Fig. 5. We note that all materials used in this actuation unit are either Ultem or PEEK (biocompatible polymers), stainless steel (which would be passivated before clinical use), or aluminum (which would
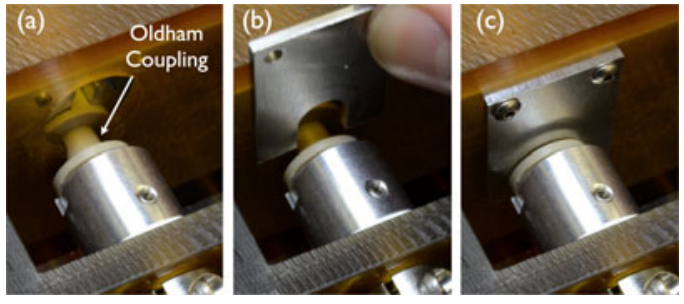

Fig. 4. Oldham couplings are used to transfer rotation of motor shafts through a tortuous path to the sterile lead screws and square shaft for translation and rotation of the cannula tubes. (a) Oldham coupling made from PEEK plastic (b) Aluminum plate is brought down over the Oldham coupling and secured in place. (c) Aluminum plate creates the tortuous path between the nonsterile bagged motor pack and the sterile robot.

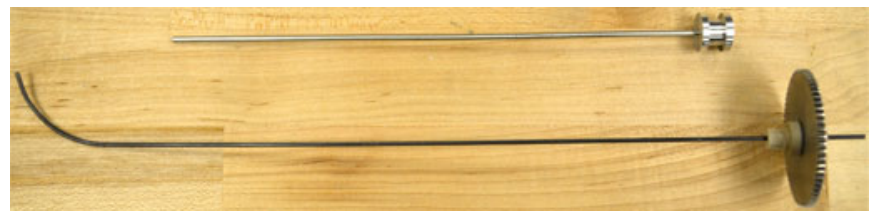

Fig. 5. Tubes are permanently attached to hubs using a biocompatible glue and can be autoclaved. The precurved aspiration tube has an additional gear attached to the hub to rotate the tube.

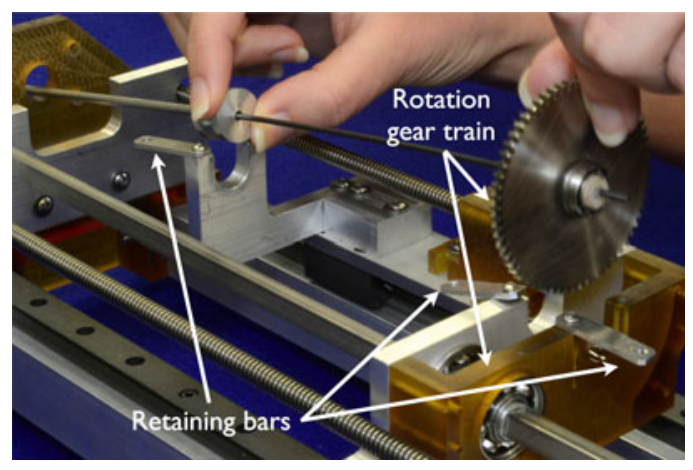

Fig. 6. Quick-release mechanism for the tubes. Either tube can be quickly released from the mechanism by removing a screw and swinging a retaining bar out of the way.

be anodized before clinical use). The trajectory guide components described in the next section are currently rapid prototype plastic, and would be replaced with a clinical-grade plastic (e.g., PEEK) before clinical use.

The system was designed to enable several aspiration tubes to be used sequentially (see Section III), so the actuation unit features the quick-release mechanism shown in Fig. 6. Removing a screw and swinging a retaining bar out of the way allows one to release a tube from the mechanism and introduce a new tube. In addition, a quick release is provided for the carriers so that in an emergency situation, the tubes can be retracted manually by disengaging the carriers from the lead screws (see Fig. 7). In an eventual clinical system, we intend to replace the three screws in this quick-release mechanism with a thumb screw approach to allow for faster operation.

\section{B. Open Loop Robot Positioning Accuracy}

To evaluate the accuracy of the robot in terms of tip position error, we recorded the position of the tip of the aspiration tube 

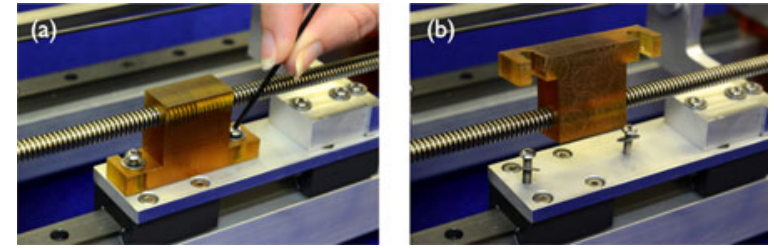

Fig. 7. Quick-release mechanism for carriers enabling them to be detached from the lead screws and manually slid backward, for manual tube retraction. The mechanism works by (a) loosening holding screws, followed by (b) swinging the threaded block away from the carrier.
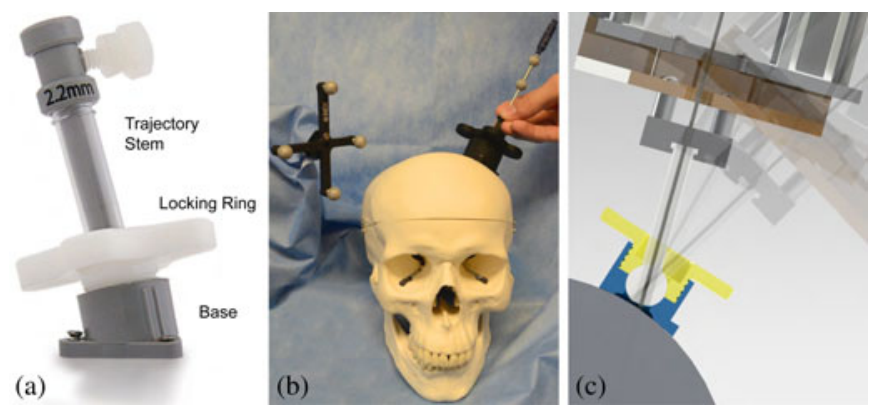

Fig. 8. Image guidance for robot-assisted ICH evacuation. (a) Commercial neuronavigation system (Navigus, Medtronic, Inc.) consists of a trajectory stem that can be securely fastened to a bone-anchored base. (b) Trajectory stem is adjusted to the desired insertion trajectory using a tracked alignment probe that enables visualization of the trajectory in registered medical images. (c) Robot system attaches to the trajectory stem.

used for the experiment in Section V-B, which was inserted through a stiff, straight outer tube, using an optical tracker and tracked point probe (Claron MicronTracker, Ontario, Canada). We used the actuation unit to move the tip of the aspiration tube to 33 evenly distributed Cartesian grid points (10-mm grid spacing) within a sphere $4 \mathrm{~mm}$ in diameter, representing a clot. The average root-mean-square error between kinematic model predictions and optical tracking data was $0.78 \mathrm{~mm}$, with a standard deviation of $0.31 \mathrm{~mm}$ and maximum error of $1.41 \mathrm{~mm}$. We note that this is the open loop accuracy of the robot, and that in a clinical implementation we envision use of imaging to servo the tip to the correct location. Hence, an open loop error is not a lower bound for overall system error.

\section{Surgical Workflow and Image-Guidance}

Our robot incorporates image-guidance using preoperatively acquired computed tomography (CT) images of the patient combined with the registration procedure employed in modern clinical image-guidance systems. In these, registration is accomplished using a surface scan of the patient's face, which is then matched to the corresponding surface in the CT image volume using a standard surface-based registration. After registration, we employ a needle alignment approach conceptually identical to that of the Navigus system (Medtronic, Inc., USA), which is illustrated in Fig. 8, where the only difference is that we have made our own version of Navigus alignment hardware via rapid prototyping, so that we can couple the trajectory stem to our robot.
To set up and align the needle, the surgeon first creates a hole in the skull, opens the dura, and attaches the base of the trajectory guide to the skull using three small titanium screws. The trajectory stem is then snapped into the base and loosely secured with the locking ring. An alignment probe (which enables visualization of the insertion trajectory on the image-guidance system, see Fig. 8(b)) is then inserted into the trajectory stem, and image guidance is used to align it (as it pivots in the base) with the desired target. The locking ring is then tightened to fix the trajectory stem in place, and the alignment probe removed. Next, the robot (attached to a passive arm) is moved into the surgical field, and the robot front plate is coupled to the trajectory stem. The active cannula then passes through the trajectory stem and into the brain, along the desired trajectory. Lastly, note that in traditional biopsy with the Navigus system, the rotation of the needle about its own axis is not important. However, it is important in our system and can be obtained via optical tracking of the robot itself, which is the reason for the tracking frame shown in Fig. 1.

\section{ASPIRATION TUBE DESIGN}

In principle, any precurvature (including those that vary with arc length) that can be straightened completely with a maximum material strain within the elastic range of nitinol (approximately $8-10 \%$ ) can be preset into the aspiration cannula via a heat treatment process. The shape and workspace of the device can be computed using the mechanics-based model described in [21] and [22]. In the future for clots with complex geometry, tubes with variable curvature may be desirable. However, as a starting point, in this paper we consider only the special case where the aspiration cannula has constant precurvature (i.e., its shape is an arc of a circle).

\section{A. Kinematics}

We parametrize the $3 \mathrm{DOF}$ of a cannula with a straight outer tube and circularly precurved inner tube using the variables $\rho_{1}$ and $\rho_{2}$ to describe the linear insertion distance of the outer straight tube and inner aspiration cannula, respectively. The angle $\alpha$ describes the axial angle of the aspiration tube and can be controlled directly by the robotic actuation unit described in the previous section. Thus, the joint space of the robot is $q=\left(\rho_{1}, \rho_{2}, \alpha\right)$. The aspiration tube is composed of an initial straight section with length $L_{s}$ followed by a planar constant curvature section with length $L_{c}$ with radius $r$ (see Fig. 9(a)). The mapping from joint space to configuration space parameters describing the curve of the robot (i.e., "arc parameters," see [30]) is as follows:

$$
\begin{aligned}
& \ell_{1}=\rho_{1} \\
& \ell_{2}= \begin{cases}\rho_{2}-\rho_{1}-L_{c} & \text { if } \rho_{2}-\rho_{1}>L_{c} \\
0 & \text { else }\end{cases} \\
& \ell_{3}= \begin{cases}L_{c} & \text { if } \rho_{2}-\rho_{1}>L_{c} \\
\rho_{2}-\rho_{1} & \text { else }\end{cases} \\
& \kappa_{3}=r^{-1} .
\end{aligned}
$$




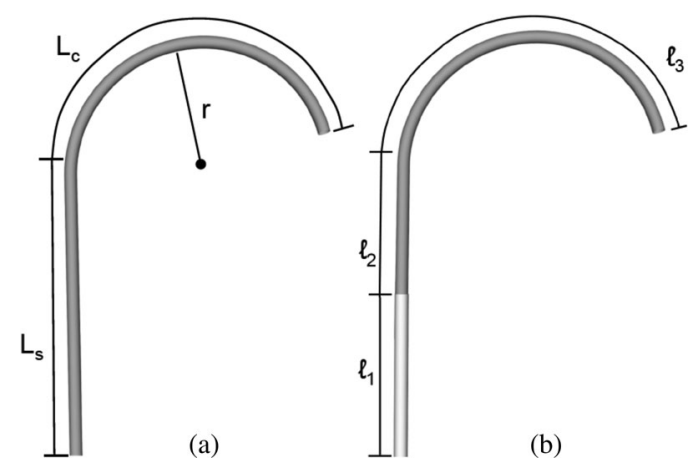

Fig. 9. (a) Each aspiration tube has a straight section with length $L_{s}$ followed by a circular curved section of length $L_{c}$ with a radius of curvature $r$. (b) When inserted into a straight outer tube (lighter colored tube that ends at $\ell_{1}$ in the image), there are three regions to model kinematically, with lengths $\ell_{1}, \ell_{2}$, and $\ell_{3}$.

These parameters define a forward kinematic mapping $T=$ $T_{\alpha} T_{12} T_{3}$, where

$$
\begin{aligned}
T_{\alpha}= & {\left[\begin{array}{cccc}
\cos \alpha & -\sin \alpha & 0 & 0 \\
\sin \alpha & \cos \alpha & 0 & 0 \\
0 & 0 & 1 & 0 \\
0 & 0 & 0 & 1
\end{array}\right] } \\
T_{12}= & {\left[\begin{array}{lllll}
1 & 0 & 0 & 0 & \\
0 & 1 & 0 & 0 \\
0 & 0 & 1 & \ell_{1}+\ell_{2} \\
0 & 0 & 0 & 1
\end{array}\right] } \\
T_{3}= & {\left[\begin{array}{cccc}
1 & 0 & 0 & 0 \\
0 & \cos \left(\kappa_{3} \ell_{3}\right) & -\sin \left(\kappa_{3} \ell_{3}\right) & \frac{\cos \left(\kappa_{3} \ell_{3}\right)-1}{\kappa_{3}} \\
0 & \sin \left(\kappa_{3} \ell_{3}\right) & \cos \left(\kappa_{3} \ell_{3}\right) & \frac{\sin \left(\kappa_{3} \ell_{3}\right)}{\kappa_{3}} \\
0 & 0 & 0 & 1
\end{array}\right] . }
\end{aligned}
$$

\section{B. Tube Design Optimization}

An optimal aspiration tube results in a two-tube active cannula with a workspace that completely covers the volume of the hemorrhage. For some clots, this may not be possible with one aspiration cannula, so two or more may be used sequentially, while leaving the outer needle in place. Given that we have restricted our tube shapes to circular in this paper, the parameters available for design are the curvature and arc length of the aspiration cannula, such that a given design is defined as $d=$ $\left\{L_{c}, r\right\}$.

We begin with CT medical image data that is routinely acquired during the diagnosis of ICH. A surface model of the skull can be determined by a simple threshold, while the hemorrhage is identified via manual segmentation in 3d Slicer [31]. We then discretize the joint space $q=\left(\rho_{1}, \rho_{2}, \alpha\right)$ of the active cannula, and compute the workspace of the device by using the kinematic model for each discrete combination of joint positions. The last step is to compare the overlap of this workspace and the segmented clot. To evaluate this overlap, we must first know the entry point and entry vector of the cannula into the clot. These are determined by the neurosurgeon, who selects the burr hole point on the skull, and then verifies that the shortest path to the hemorrhage does not pass through any critical brain structures.

We now describe the formulation of a volumetric objective function quantifying the coverage of a given clot achievable by a given cannula design. The surface model of the hemorrhage is converted into a set of isotropic voxels. We use a binary voxel representation to differentiate between voxels inside and outside the hemorrhage. We then use as our objective function for clot coverage by a given design $(d)$ the percentage of the total clot volume that is accessible to the tip of the cannula. The portion of the cannula workspace volume that lies within the hematoma $V(d)$ is generated for each $d$ by discretizing the joint space of the robot with $1 \mathrm{~mm}$ translational and $1^{\circ}$ rotational increments and computing the cannula tip position for each combination of joint values. We also perform a secondary check that keeps only configurations in which the entire curve of the aspiration tube is within the clot. The voxels that contain remaining tip points are labeled as covered voxels, while others are labeled as uncovered. Finally, the percentage of the clot covered is computed by dividing the total number of clot voxels by the number of covered voxels. Our current implementation (C++, Intel Core i7 2600) requires on average less than $1 \mathrm{~min}$ to calculate the covered clot volume for one design $d$ and a given entry path. We expect a speed-up through parallelization to a few seconds per tube design evaluation.

\section{Patient-Specific Optimization CASE Studies}

We conducted a retrospective, IRB approved study using seven CT datasets from patients with ICH previously treated at Vanderbilt University Medical Center. Surface models of each skull and hemorrhage are shown in Fig. 10. For each case a neurosurgeon selected a desired entry path, and for each path all possible aspiration tube sets were evaluated using the objective function in the previous section. Tube parameters were as follows:

1) Aspiration tubes were considered to have an outer diameter of $1.14 \mathrm{~mm}$ and an inner diameter of $0.91 \mathrm{~mm}$, matching the experimental tubes used in Section V-B.

2) A $10 \%$ recoverable strain threshold was used.

3) The curved section length $L_{c}$ was first discretized in 5mm steps starting at $10 \mathrm{~mm}$. Then, the same procedure was used with a finer resolution of $2 \mathrm{~mm}$. Tables I-III show the best choices across both discretizations.

4) Minimum and maximum radii of curvature were 6.4 and $150 \mathrm{~mm}$, respectively, with $2.5 \mathrm{~mm}$ discretization within this range.

For selection of the optimal aspiration tube(s), three scenarios of use are now considered.

\section{A. Scenario 1: Single Aspiration Tube}

In this first scenario, we require the aspiration tube to remain within the clot at all times, and consider that only one aspiration tube is available to be deployed through the straight outer tube. For each patient case, we computed the tube curvature and arc 


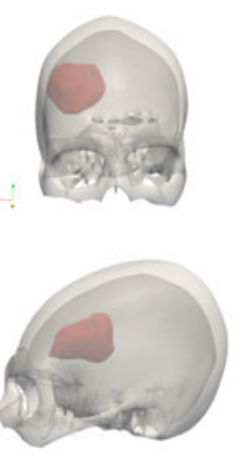

1
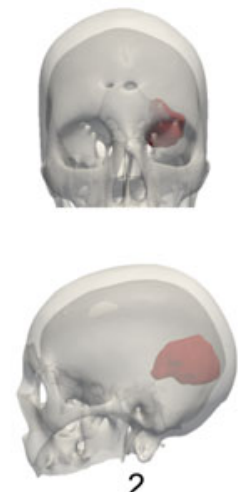

2
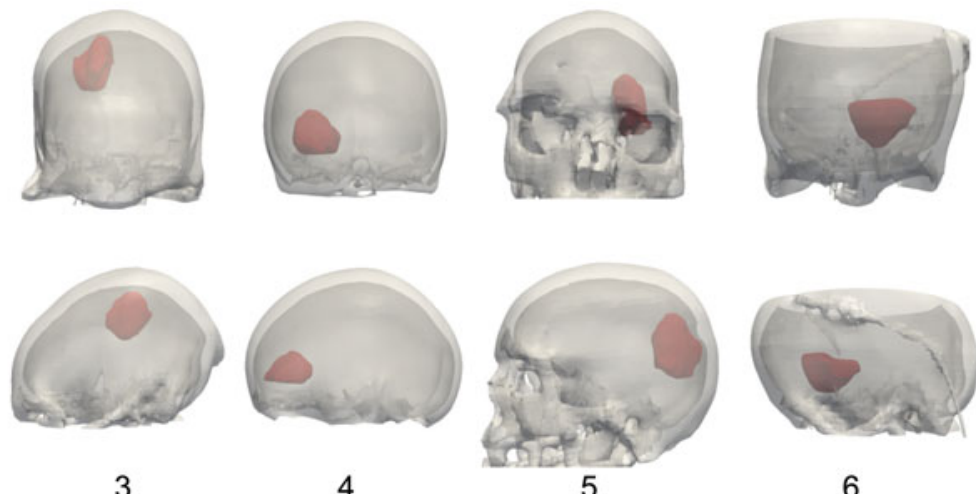

5

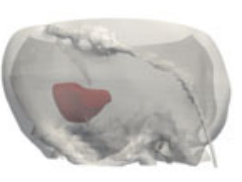

6
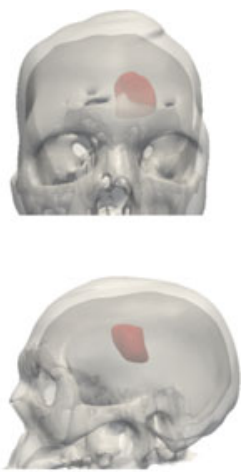

7

Fig. 10. Seven cases of patients with an $\mathrm{ICH}$ (hemorrhage shown in red).

TABLE I

SUMMARY OF OPTIMAL ASPIRATION TUBES FOR SCENARIO 1

\begin{tabular}{c|cc|c}
\hline Case & $l_{c}$ & $r$ & $f^{*}$ \\
\hline 1 & 108 & 17.19 & $66 \%$ \\
2 & 85 & 13.53 & $71 \%$ \\
3 & 50 & 10.46 & $85 \%$ \\
4 & 75 & 11.94 & $68 \%$ \\
5 & 70 & 11.14 & $88 \%$ \\
6 & 55 & 13.75 & $61 \%$ \\
7 & 65 & 10.35 & $75 \%$ \\
\hline
\end{tabular}

TABLE II

SUMMARY OF OPTIMAL ASPIRATION TUBES FOR SCENARIO 2

\begin{tabular}{c|cc|c|c}
\hline Case & $L_{c}$ & $r$ & $t_{d}$ & $f^{*}$ \\
\hline 1 & 120 & 19.10 & 14.90 & $93 \%$ \\
2 & 80 & 17.73 & 10.59 & $94 \%$ \\
3 & 110 & 17.51 & 9.09 & $99 \%$ \\
4 & 80 & 12.73 & 3.42 & $88 \%$ \\
5 & 80 & 12.73 & 7.02 & $98 \%$ \\
6 & 60 & 29.63 & 15.00 & $98 \%$ \\
7 & 60 & 44.63 & 15.00 & $100 \%$ \\
\hline \multicolumn{5}{|c}{ The upper bound for $t_{d}$ as Set at $15 \mathrm{~mm}}$.
\end{tabular}

TABLE III

Scenario 3: Sequential USE of Two Aspiration Tubes With COMBINED COVERAGE $f^{*}$

\begin{tabular}{c|ccc|ccc|c}
\hline & \multicolumn{3}{|c|}{ Tube 1 } & \multicolumn{3}{|c|}{ Tube 2 } & \\
Case & $L_{c}$ & $r$ & $f$ & $L_{c}$ & $r$ & $f$ & $f^{*}$ \\
\hline 1 & 70 & 16.14 & $67 \%$ & 60 & 9.55 & $57 \%$ & $86 \%$ \\
2 & 60 & 17.05 & $66 \%$ & 55 & 8.75 & $62 \%$ & $87 \%$ \\
3 & 45 & 12.16 & $82 \%$ & 40 & 6.37 & $65 \%$ & $94 \%$ \\
4 & 80 & 12.73 & $67 \%$ & 45 & 7.16 & $60 \%$ & $87 \%$ \\
5 & 60 & 12.05 & $87 \%$ & 45 & 7.16 & $67 \%$ & $96 \%$ \\
6 & 60 & 14.54 & $60 \%$ & 55 & 8.75 & $49 \%$ & $78 \%$ \\
7 & 40 & 11.37 & $73 \%$ & 40 & 6.36 & $63 \%$ & $90 \%$ \\
\hline
\end{tabular}

length that maximizes coverage of the hematoma $(f)$

$$
f^{*}=\arg \max f(d)
$$

with results summarized in Table I . While this level of coverage exceeds the $25-50 \%$ minimum coverage target mentioned in the introduction, we also went on to consider two other scenarios of use, in order to provide additional options for the neurosurgeon if increased coverage is desired.

\section{B. Scenario 2: Single Aspiration Tube With Brain Deformation}

We now relax the requirement that the entire curved tube remains inside the clot at all times, and instead permit small lateral deflection at the surface of the clot up to some threshold $t_{d}$ as shown in Fig. 11. By doing this, we can significantly increase

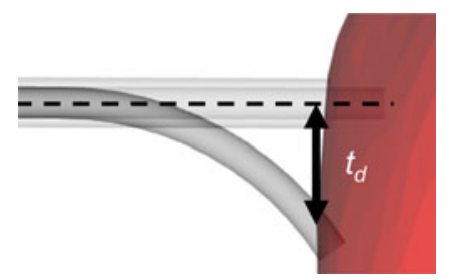

Fig. 11. This figure illustrates the tissue deflection threshold $t_{d}$, which applies only to Scenario 2 (Section IV-B). The quantity $t_{d}$ is the maximum distance between the entry axis of the device and the point at which the curved tube enters the clot. It is a surgeon-specified parameter.

the volume of clot accessible to a single constant curvature aspiration tube as the results in Table II illustrate (here we used a maximum $t_{d}$ of $15 \mathrm{~mm} ; t_{d}$ is in general based on the preference of the specific surgeon using the system, and if it is set to zero, then Scenario 2 is identical to Scenario 1). Note also that one can achieve a configuration like that shown in Fig. 11 without cutting brain tissue by first deploying the aspiration tube within the clot, and then retracting the straight outer tube and the aspiration tube simultaneously.

\section{Scenario 3: Two Aspiration Tubes in Succession}

In this scenario, we sought to maintain the high coverage achieved in Scenario 2 while eliminating the need for brain deflection (i.e., keeping the aspiration tube completely within the hematoma as was done in Scenario 1). We achieve this by selecting two aspiration tubes with different curvatures, which will be sequentially used. After the first tube has evacuated all of the clot it is able to reach, it is removed with the outer straight tube remaining in place in the patient's brain, such that the system maintains registration, and a second aspiration tube is introduced to remove additional clot material. The goal is to choose the parameters of the two tubes simultaneously, such that the overall volume of the clot removed is maximized

$$
f^{*}=\arg \max \left(f\left(d_{1}\right) \cap f\left(d_{2}\right)\right) .
$$

Results are shown in Table III.

The similarity in curvatures of both tubes across all cases is noteworthy, as is the high overall volume of clot removed. To determine how well a single set of two tubes could work across all patients, we reran the optimization seeking to maximize the average coverage across all patients. This resulted in a first 
TABLE IV

SCENARIO 4: Sequential USE of FiVe PRESELECTEd Tubes AND INFLUENCE OF ENTRY PATH PERTURBATION

\begin{tabular}{c|c|c}
\hline Case & $\begin{array}{c}\text { Optimal Entry Path } \\
f^{*}\end{array}$ & $\begin{array}{c}\text { Perturbed Entry Paths } \\
\min f^{*}\end{array}$ \\
\hline 1 & $92 \%$ & $60 \%$ \\
2 & $94 \%$ & $85 \%$ \\
3 & $98 \%$ & $97 \%$ \\
4 & $96 \%$ & $57 \%$ \\
5 & $100 \%$ & $95 \%$ \\
6 & $92 \%$ & $32 \%$ \\
7 & $95 \%$ & $95 \%$ \\
\hline
\end{tabular}

tube with $L_{c}=58 \mathrm{~mm}, r=13.23 \mathrm{~mm}$ and a second tube with $L_{c}=40 \mathrm{~mm}, r=6.37 \mathrm{~mm}$. This tube set enables an average coverage of $79 \%$ across all patients, with a minimum of $60 \%$ and a maximum of $95 \%$.

\section{Scenario 4: Discrete Tube Set}

In this scenario, we evaluated the performance of a fixed set of five tubes (radii of curvature: $6,8.5,11,13.5$, and $16 \mathrm{~mm}$ ), where the radii were chosen to span the optimal tube curvatures of Scenario 3. The five tubes used in sequence are able to remove an average of $95 \%$ of each clot in our patient dataset (see column 2 of Table IV).

Noting that there will be some uncertainty in burr hole placement and also in targeting the desired clot entry point, we performed a perturbation study. In considering the level of error that can be expected in clinical use, note that the accuracy of the Navigus in targeting locations within the skull has been experimentally found to be $1 \mathrm{~mm}$ with a standard deviation of $0.28 \mathrm{~mm}$ [32], [33]. Also note that the surgeon will have access to image-guidance when selecting the burr hole location, and thus, in principle should be able to place the burr hole at the desired location approximately as accurately as internal skull points can be targeted (the same image-guidance system being used for both purposes). To establish a conservative level of error, we went out a little over three standard deviations, considering a maximum burr hole error of $2.5 \mathrm{~mm}$ and a maximum clot entry point error of $2 \mathrm{~mm} .{ }^{1}$ Worst case needle angular misalignment within these bounds occurs when both burr hole error and clot entry error are simultaneously deviate maximally from the planned locations, and do so in the worst possible direction (worst both with respect to one another, and with respect to clot geometry). ${ }^{2}$

We generated 25 perturbation cases for each of our seven patient cases by considering all combinations of five evenly angularly distributed points at a radial distance of $2.5 \mathrm{~mm}$ on the skull surface around planned burr hole location and five evenly angularly distributed points at a radial distance of $2 \mathrm{~mm}$ around the planned clot entry point. Column 3 of Table IV shows the minimum clot volume coverage across all patient and perturbation cases. It can be concluded from this study that if surgeons

\footnotetext{
${ }^{1}$ Burr hole error was set slightly higher than the clot targeting error to account for the fact that the surgeon may be slightly more careful in internal point targeting than in burr hole placement.

${ }^{2}$ We note that since the errors are likely Gaussian in nature, it is statistically unlikely that both errors would be maximal simultaneously (let alone in the worst possible direction), but considering such a case provides a useful lower bound on worst case clot coverage.
}

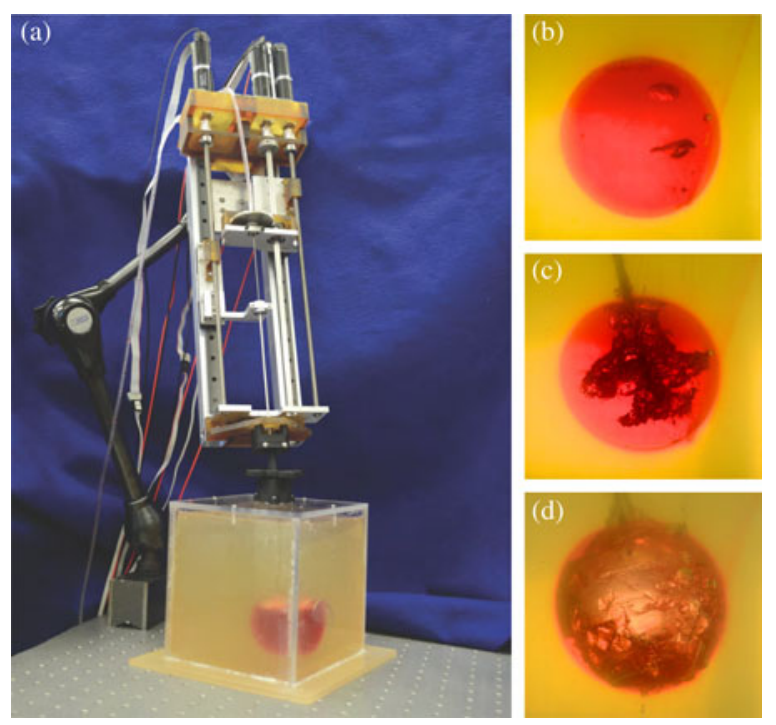

Fig. 12. Phantom experiment. (a) Robot is attached to the passive arm and secured to the trajectory guide, which is mounted onto the top of an acrylic box. The active cannula is deployed to the clot and the aspiration tube is used to debulk the clot. (b) Blood clot is shown prior to beginning the experiment, (c) progress midway through the removal experiment, and (d) the same area after aspiration.

are correct in their estimate that decompression benefit begins when $25-50 \%$ of the clot is removed, it is statistically improbable that a small number of discrete tubes will be incapable of accessing the requisite geometry.

\section{IN VITRO DEMONSTRATIONS}

\section{A. In Vitro Phantom Experiment}

To explore the practical feasibility of robot-assisted ICH evacuation using a single tube with allowed tissue deflection (see Section IV-B), we conducted a phantom experiment. The aspiration tube used had a straight section with $L_{s}=260 \mathrm{~mm}$ followed by a section with constant curvature of $r=30.3 \mathrm{~mm}$ with $L_{c}=55 \mathrm{~mm}$. The aspiration tube had an outer diameter of $1.75 \mathrm{~mm}$ and a wall thickness of $0.3 \mathrm{~mm}$. The outer tube had an outer diameter of $3.2 \mathrm{~mm}$ and a wall thickness of $1 \mathrm{~mm}$.

The experiment was conducted using a gelatin phantom (see Fig. 12(a)). The clear tissue in the phantom simulated regular brain tissue and was made from $10 \%$ by weight Knox gelatin (Kraft Foods Global, Inc., USA), and the red tissue that simulated the clot was made from Jell-O gelatin. This made the hematoma softer than the surrounding brain tissue. This phantom is similar to the one used in [19]. The hemorrhage was approximately spherical with a $63.5-\mathrm{mm}$ diameter.

The trajectory stem was aligned with the clot and secured using the locking ring. The robot was then affixed to the passive arm and attached to the trajectory stem as discussed in Section II-C. The outer tube was then inserted into the clot, with the inner tube retracted fully inside it, and the tubes were then used to evacuate the clot. Motion planning was conducted manually by the experimenter who visually observed the debulking process through the wall of the phantom and input new desired target locations to the robot manually using the computer keyboard. The robot was able to remove $92 \%$ of the clot material, 


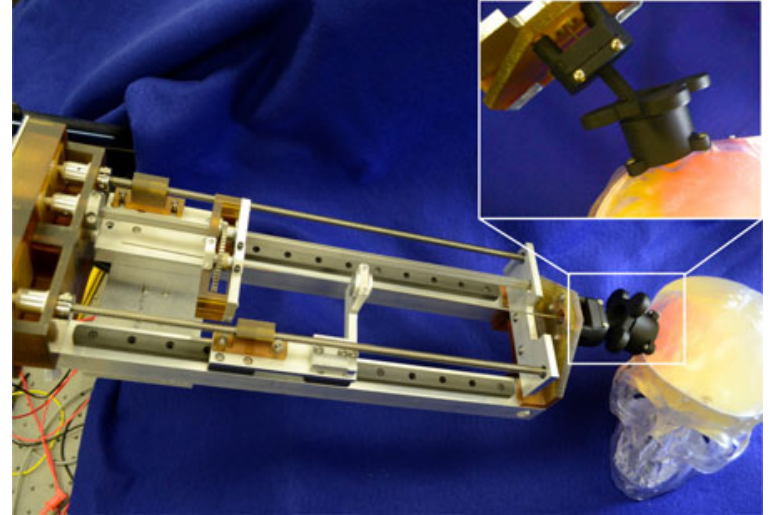

Fig. 13. In vitro skull experiment. The robot was aligned using the trajectory stem and the aspirator was connected to the end of the inner tube. The clot was then removed by moving the tip of the cannula within the clot while applying suction.

determined by initially measuring the amount of red gelatin used. The surface of the clot was visually inspected for positive margins and none were detected. The residual red gelatin at the end of the experiment was scooped out and weighed. The achieved results are similar to the $99 \%$ theoretical coverage of this clot using Scenario 2 from the previous section, and the system could have removed more material at the clot-brain interface had we been less concerned with damaging healthy brain tissue. Fig. 12 shows the experimental setup and before, during, and postexperiment photos of the clot.

\section{B. In Vitro Skull Experiment}

We next performed an experiment in vitro using an anatomically correct skull model and one of the patient clots from Section IV. The goal was to experimentally demonstrate the system under conditions like those in patient case 1 of Scenario 3 from Section III. To replicate the geometry of patient case 1, we used a two-piece, semitransparent plastic skull (A20/T, 3B Scientific, USA), which we filled with gelatin. The segmented clot from patient case 1 was made using a rapid prototyping machine, and we also prototyped the negative form of the clot. A thin acrylic sheet was laser cut to fit in between the two halves of the skull, and a hole was laser cut in the sheet such that the prototyped clot would sit at the correct height and orientation in the skull. The plate and skull were assembled and sealed using silicone before the liquid gelatin was introduced. The net result was a two-piece gelatin mold with an empty cavity for the prototyped clot. We then used the negative form of the clot to mold a Jell-O model of the clot, and added barium at a mixture of $0.5 \mathrm{~g} / \mathrm{ml}$ to enable visualization of the clot in CT.

We shape set the nitinol tubes used in this experiment using heat treatment. Although we attempted to achieve the curvatures listed on Table III for patient case 1, the tubes relaxed slightly as they were removed from the fixture that held them in the oven, such that the measured radii of curvature were 19.8 and $12.6 \mathrm{~mm}$. The robot was aligned with the entry path selected by an experienced neurosurgeon (see Fig. 13). Because the skull was not completely transparent, the top of the skull
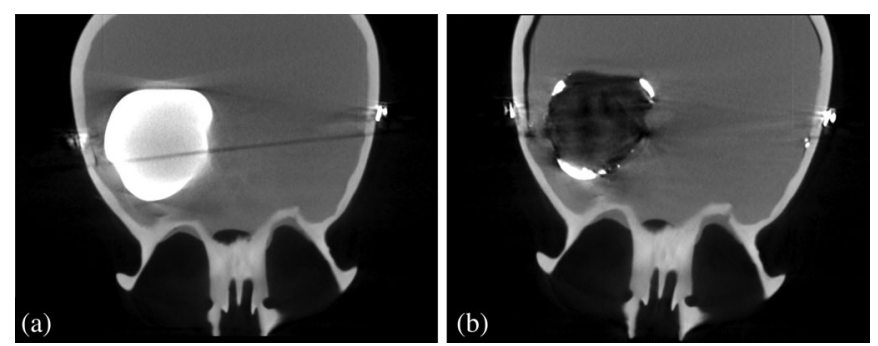

Fig. 14. Slices from CT scans of the phantom (a) before aspiration and (b) after aspiration. The robotic system was able to remove $83.1 \%$ of the clot.

was removed after the robot was aligned to enable visualization of the tubes in the clot. Motion planning, robot position commands, and determination of the removed clot volume were implemented the same way as in the previous experiment. The robot was able to remove $83.1 \%$ of the clot (see Fig. 14), which was measured using the same procedure as in the previous experiment. Based on the curvatures of the experimental tubes, the expected clot removal percentage was $80.6 \%$. The fact that the experimental results slightly exceeded the theoretical prediction can be attributed to minor tissue deformation as suction is applied, which brings more material within reach of the cannula tip. We note that deformation is likely to be present in human brain tissue, so the theoretical percentages given in this paper (which consider only rigid geometry) may be conservative. See the conclusion for further discussion of the future work that we envision to compensate for deformation effects.

\section{CONCLUSION AND DISCUSSION}

In this paper, we have explored the feasibility of a new approach to debulking $\mathrm{ICH}$ clots from within using a procedure with invasiveness comparable to a standard brain biopsy. As first steps toward clinical deployment of the technique, we have described the design of a sterilizable robotic actuation unit, several methods for tube curvature design, and several deployment scenarios. A retrospective simulation study on patient CT images demonstrated that one or two circularly curved tubes are sufficient to cover a high percentage of clot geometry across all subjects in our dataset. In vitro experiments illustrated our proposed approach. As mentioned in the introduction, surgeons estimate that clinical benefit will begin to occur for patients if $25-50 \%$ of the clot can be removed. Our simulations and in vitro experiments indicate that percentages significantly higher than this are accessible with even simple constant curvature cannulas.

Many challenges remain before our proposed approach can be implemented clinically. Since brain deformation may be significant in ICH removal, we believe that intraoperative imaging and brain deformation modeling (together with motion planning algorithms that leverage both) will be important to ensure that the cannula does not accidentally leave the clot and impinge on brain tissue as the clot changes shape. An additional layer of safety in a human case can be obtained by being less aggressive than we were in our in vitro experiments and not attempting to remove $100 \%$ of the clot; one could intentionally leave a margin of clot around the edges, debulking the middle of the clot 
only. Furthermore, while we believe that aspiration will locally remove clot material, this remains to be tested, and we may find that morcellation will ultimately be necessary. Fortunately, it is possible to include a morcellator on the tip of a concentric tube robot as was shown in [34].

In summary, in this paper, we have pursued early feasibility studies on a new concept for ICH decompression that may eventually bring the benefits of less invasive decompression to many patients. Our hope is that our new decompression approach will eventually save the lives of some of the $40 \%$ of patients who currently die from $\mathrm{ICH}$, and also that it may eventually reduce the instances of brain damage in those patients who survive.

\section{ACKNOWLEDGMENT}

The authors acknowledge insights on biocompatibility, safety, and usability features provided by E. Clif Burdette and his team at Acoustic MedSystems, Inc., which inspired aspects of the design presented in Section II-A. The content is solely the responsibility of the authors and does not necessarily represent the official views of the National Science Foundation.

\section{REFERENCES}

[1] C. J. van Asch, M. J. Luitse, G. J. Rinkel, I. van der Tweel, A. Algra, and C. J. Klijn, "Incidence, case fatality, and functional outcome of intracerebral haemorrhage over time, according to age, sex, and ethnic origin: A systematic review and meta-analysis," Lancet Neurol., vol. 9, no. 2, pp. 167-176, 2010.

[2] R. A. Kaya, O. Türkmenolu, I. M. Ziyal, T. Dalkiliç, Y. Ahin, and Y. Aydin, "The effects on prognosis of surgical treatment of hypertensive putaminal hematomas through transsylvian transinsular approach," Surg. Neurol., vol. 59, no. 3, pp. 176-183, 2003.

[3] G. Maira, C. Anile, C. Colosimo, and G. F. Rossi, "Surgical treatment of primary supratentorial intracerebral hemorrhage in stuporous and comatose patients," Neurol. Res., vol. 24, no. 1, pp. 54-60, 2002.

[4] J. M. Murthy, G. V. Chowdary, T. V. Murthy, P. S. Bhasha, and T. J. Naryanan, "Decompressive craniectomy with clot evacuation in large hemispheric hypertensive intracerebral hemorrhage," Neurocritical Care, vol. 2, no. 3, pp. 258-262, 2005.

[5] S. H. Tan, P. Y. Ng, T. T. Yeo, S. H. Wong, P. L. Ong, and N. Venketasubramanian, "Hypertensive basal ganglia hemorrhage: A prospective study comparing surgical and nonsurgical management," Surg. Neurol., vol. 56, no. 5, pp. 287-292, 2001.

[6] Y. Zuo, G. Cheng, D.-K. Gao, X. Zhang, H.-N. Zhen, W. Zhang, and S.-C. Xiao, "Gross-total hematoma removal of hypertensive basal ganglia hemorrhages: A long-term follow-up," J. Neurol. Sci., vol. 287, no. 1-2, pp. 100-104, 2009.

[7] L. Elijovich, P. V. Patel, and J. C. Hemphill, "Intracerebral hemorrhage," Seminars Neurol., vol. 28, no. 5, pp. 657-667, 2008.

[8] C.-H. Chen, H.-T. Lee, C.-C. Shen, and M.-H. Sun, "Aspiration of hypertensive intracerebral hematoma with frameless and fiducial-free navigation system: Technical note and preliminary result," Stereotactic Functional Neurosurg., vol. 86, no. 5, pp. 288-291, 2008.

[9] C.-C. Chen, D.-Y. Cho, C.-S. Chang, J.-T. Chen, W.-Y. Lee, and H.-C. Lee, "A stainless steel sheath for endoscopic surgery and its application in surgical evacuation of putaminal haemorrhage," J. Clin. Neurosci., vol. 12, no. 8, pp. 937-940, 2005.

[10] N. Etminan, K. Beseoglu, B. Turowski, H. J. Steiger, and D. Hanggi, "Perfusion CT in patients with spontaneous lobar intracerebral hemorrhage: Effect of surgery on perihemorrhagic perfusion," Stroke, vol. 43, no. 3, pp. 759-763, 2012.

[11] D. W. Newell, M. M. Shah, R. Wilcox, D. R. Hansmann, E. Melnychuk, J. Muschelli, and D. F. Hanley, "Minimally invasive evacuation of spontaneous intracerebral hemorrhage using sonothrombolysis," J. Neurosurg., vol. 115, no. 3, pp. 592-601, 2011.

[12] C. M. Miller, P. Vespa, J. L. Saver, C. S. Kidwell, S. T. Carmichael, J. Alger, J. Frazee, S. Starkman, D. Liebeskind, V. Nenov, R. Elashoff, and N. Martin, "Image-guided endoscopic evacuation of spontaneous intracerebral hemorrhage," Surg. Neurol., vol. 69, no. 5, pp. 441-446, 2008

[13] Y. S. Kwoh, J. Hou, E. A. Jonckheere, and S. Hayati, "A robot with improved absolute positioning accuracy for CT guided stereotactic brain surgery," IEEE Trans. Biomed. Eng., vol. 35, no. 2, pp. 153-160, Feb. 1988.

[14] D. F. Louw, T. Fielding, P. B. McBeth, D. Gregoris, P. Newhook, and G. R. Sutherland, "Surgical robotics: A review and neurosurgical prototype development," Neurosurg., vol. 54, no. 3, pp. 525-536, 2004.

[15] R. Taylor, "A perspective on medical robotics," Proc. IEEE, vol. 94, no. 9, pp. 1652-1664, Sep. 2006

[16] G. Dogangil, B. L. Davies, and F. Rodriguez y Baena, "A review of medical robotics for minimally invasive soft tissue surgery," Proc. Inst. Mech. Eng. Part H: J. Eng. Med., vol. 224, no. 5, pp. 653-679, 2010.

[17] J. A. Engh, G. Podnar, D. Kondziolka, and C. N. Riviere, "Toward effective needle steering in brain tissue," in Proc. Int. Conf. IEEE Eng. Med. Biol. Soc., Aug./Sep. 2006, vol. 1, pp. 559-562.

[18] R. J. Webster III, J. S. Kim, N. J. Cowan, G. S. Chirikjian, and A. M. Okamura, "Nonholonomic modeling of needle steering," Int. J. Robot. Res., vol. 25, no. 5-6, pp. 509-526, 2006.

[19] T. M. Goradia, R. H. Taylor, and L. M. Auer, "Robot-assisted minimally invasive neurosurgical procedures: First experimental experience," in First Joint Conf. Vision, Virtual Reality, Robotics Med. Med. Robot. Comput.Assisted Surgery, 1997, pp. 319-322.

[20] J. M. Simard, "Plenary lectures: Problems in neurosurgery-a rich environment for engineers," in Proc. IEEE Int. Conf. Biomed. Robot. Biomechatron., 2012, p. L. DOI: 10.1109/BioRob.2012.6290953.

[21] P. E. Dupont, J. Lock, B. Itkowitz, and E. Butler, "Design and control of concentric-tube robots," IEEE Trans. Robot., vol. 26, no. 2, pp. 209-225, Apr. 2010.

[22] D. C. Rucker, B. A. Jones, and R. J. Webster III, "A geometrically exact model for externally loaded concentric-tube continuum robots," IEEE Trans. Robot., vol. 26, no. 5, pp. 769-780, Oct. 2010.

[23] T. Anor, J. R. Madsen, and P. E. Dupont, "Algorithms for design of continuum robots using the concentric tubes approach: A neurosurgical example," in Proc. IEEE Int. Conf. Robot. Autom., May 2011, pp. 667-673.

[24] C. Bedell, J. Lock, A. Gosline, and P. E. Dupont, "Design optimization of concentric tube robots based on task and anatomical constraints," in Proc. IEEE Int. Conf. Robot. Autom., May 2011, pp. 398-403.

[25] J. Burgner, P. J. Swaney, D. C. Rucker, H. B. Gilbert, S. T. Nill, P. T. Russell, K. D. Weaver, and R. J. Webster, "A bimanual teleoperated system for endonasal skull base surgery," in Proc. IEEE/RSJ Int Conf. Intell. Robot. Syst., Sep. 2011, pp. 2517-2523.

[26] J. Burgner, H. B. Gilbert, and R. J. Webster III, "On the computational design of concentric tube robots: Incorporating volume-based objectives," in Proc. IEEE Int. Conf. Robot. Autom., 2013.

[27] P. J. Swaney, J. Burgner, R. A. Lathrop, H. B. Gilbert, K. D. Weaver, and R. J. Webster III, "Minimally-invasive intracerebral hemorrhage removal using an active cannula," in Proc. IEEE Int. Conf. Robot. Autom., 2013.

[28] C. M. Graves, A. Slocum, R. Gupta, and C. J. Walsh, "Towards a compact robotically steerable thermal ablation probe," in Proc. IEEE Int. Conf Robot. Autom., May 2012, pp. 709-714.

[29] J. Burgner, P. J. Swaney, T. L. Bruns, M. S. Clark, D. C. Rucker E. C. Burdette, and R. J. Webster, "An autoclavable steerable cannula manual deployment device: Design and accuracy analysis," J. Med. Devices, vol. 6, no. 4, 2012

[30] R. J. Webster III and B. A. Jones, "Design and kinematic modeling of constant curvature continuum robots: A review," Int. J. Robot. Res., vol. 29, no. 13, pp. 1661-1683, 2010.

[31] (2012). 3d Slicer. [Online]. Available: http://www.slicer.org

[32] M. Mcdonald, N. Konyer, and M. J. Bronskill, "MRl-guided neurosurgery: Accuracy of the navigus trajectory guide," Int. Soc. Magn. Resonace Med 10, vol. 94, no. 1, p. 2075, 2002.

[33] A. Quiñones Hinojosa, M. L. Ware, N. Sanai, and M. W. McDermott, "Assessment of image guided accuracy in a skull model: Comparison of frameless stereotaxy techniques vs. frame-based localization," J. Neurooncol., vol. 76, no. 1, pp. 65-70, 2006.

[34] A. H. Gosline, N. V. Vasilyev, A. Veeramani, M. Wu, G. Schmitz, R. Chen, V. Arabagi, P. J. del Nido, and P. E. Dupont, "Metal MEMS tools for beating-heart tissue removal," in Proc. IEEE Int. Conf. Robot. Autom., May 2012, pp. 1921-1926.

Authors's photographs and biographies not available at the time of publication. 